\title{
High-resolution optical coherence tomography Application in dermatology
}

\author{
Arnaud Dubois \\ Laboratoire Charles Fabry, Institut d'Optique Graduate School, Université Paris-Sud, 91127 Palaiseau Cedex, France \\ DAMAE Medical, 28 rue de Turbigo, 75003 Paris, France \\ arnaud.dubois@institutoptique.fr
}

\begin{abstract}
Advances in optical coherence tomography (OCT) for application in dermatology are reported. Full-field OCT and line-field OCT are compared in terms of spatial resolution, penetration and acquisition rate. In vivo imaging of skin lesions is demonstrated with good similarity to histology. (C) 2019 Arnaud Dubois

OCIS codes: (110.4500) Optical coherence tomography; (170.4500) Optical coherence tomography; (170.3880) Medical and biological imaging.
\end{abstract}

\section{Introduction}

Optical coherence tomography (OCT) is an interferometric optical technique for cross-sectional imaging of biological tissues in situ and in real-time. Imaging at high spatial resolution using OCT is of particular interest for early detection of morphological changes in tissues associated with the development of tumors, notably in dermatology. The axial resolution of OCT images is governed by the temporal coherence of the detected light. Axial resolutions of $\sim 1 \mu \mathrm{m}$ have been achieved using broadband light sources such as mode-locked lasers and supercontinuum lasers. The transverse resolution of OCT images depends on how the light beam is focused on the sample. In frequency-domain OCT (FD-OCT), the sample reflectivity profile as a function of depth is acquired in parallel, which limits how highly the beam can be focused to preserve a sufficient depth of field. Several approaches have been investigated to minimize the resulting limitation of the transverse resolution in FD-OCT, including the illumination of the sample with a Bessel beam, the use of phase masks, or the use of acousto-optics. Computational methods have also been proposed, including interferometric synthetic aperture microscopy and digital refocusing. Another approach consists of combining several B-scan images acquired at different depths. Despite these advances, however, the transverse resolution in FD-OCT is still limited to a few micrometers. In time-domain OCT (TDOCT), the sample reflectivity profile as a function of depth is acquired sequentially by scanning the sample depth. In TD-OCT, the focus can theoretically be adjusted while the imaging depth is scanned. In practice, however, dynamic focusing is challenging since a tracking speed on the order of a few $\mathrm{m} / \mathrm{s}$ and a repetition rate in the kilohertz range are needed.

\section{Full-field OCT}

Full-field optical coherence tomography (FF-OCT) was introduced a few years ago as an alternative to conventional TD-OCT to obtain en face tomographic images without transverse scanning [1-3]. FF-OCT has been applied to high resolution ( $\sim 1 \mu \mathrm{m}$ at $\sim 700 \mathrm{~nm}$ center wavelength) non-invasive imaging of various semi-transparent samples [4-5]. The conventional experimental arrangement of FF-OCT is based on a white-light interference microscope in the Linnik configuration, i.e. a Michelson-type interferometer with a microscope objective placed in each arm. A halogen lamp or a broadband light emitting diode (LED) provides uniform illumination of the microscope objective fields with low coherence light. A low reflectivity mirror is placed in the reference arm of the interferometer in the focal plane of the microscope objective. An area camera acquires the interferometric images delivered by the microscope. Immersion microscope objectives are employed to minimize the mismatch between the coherence plane and the objective focal plane and to minimize the mismatch of optical dispersion in the interferometer arms [6]. The signal in FF-OCT - the amplitude of the acquired interferometric signal, i.e. the fringe envelope - is obtained by phase-shifting interferometry. The method consists of introducing a time-modulation of the relative phase between the reference and sample waves and acquiring several phase-shifted interferometric images. The phase-shift is generated either by the displacement of the reference mirror attached to a piezoelectric actuator or by the scan of the depth in the sample. Each tomographic image corresponds to an image of the reflecting/backscattering structures of the sample located in a "slice" perpendicular to the optical axis (an en face image). The width of this "slice", equal to the temporal coherence length of the detected light, defines the axial imaging resolution. The tomographic images can be produced at a maximum rate equal to a fraction of the camera frame rate depending on the number of interferometric images required in the phase-shifting algorithm. However, in practice it is necessary to accumulate 
several interferometric images to improve the detection sensitivity. The en face tomographic images are then produced at a rate on the order of a few Hertz. B-scan imaging using FF-OCT requires the acquisition of a stack of en face images, which may take several seconds. B-scan imaging using FF-OCT is usually not applicable to in vivo studies.

\section{Line-field OCT}

Line-field optical coherence tomography (LF-OCT), also referred to as line-field confocal optical coherence tomography (LC-OCT), is a recent evolution of FF-OCT with line illumination and line detection [7]. Since multiple A-scans are acquired in parallel using a line camera, only one scan (depth-scan) is required to produce a B-scan image. The scan of the depth can thus be slower compared to conventional TD-OCT, without increasing the image acquisition time, which makes dynamic focusing easier. Moreover, line illumination and detection, combined with the use of microscope objectives with relatively high numerical aperture (NA), provides a confocal gate that minimizes the amount of unwanted scattered light detected by the camera. In LF-OCT, the amount of light incident onto the photodetector that does not contribute to the interference is thus significantly reduced compared to FF-OCT [8]. This confers to LF-OCT a significant advantage over FF-OCT in terms of imaging penetration depth. LF-OCT is thus similar to FD-OCT in terms of imaging penetration in scattering tissues such as skin. Moreover, B-scan imaging can be considerably faster with LF-OCT compared to FF-OCT due to the possibility of using both a spatially coherent light source with much higher brightness and a high-speed line camera.

In our recent LF-OCT prototype, the acquired interferometric data are processed using a Fourier transform method to extract the fringe envelope and generate an intensity-based tomographic image. The B-scan images, after being appropriately rescaled, are displayed in real-time (10 frame/s). By using a supercontinuum laser as the light source and balancing the optical dispersion in the interferometer arms, the axial resolution of LF-OCT images is comparable to the best axial resolution achieved by OCT at $\sim 800 \mathrm{~nm}$ center wavelength. An isotropic spatial resolution of $\sim 1 \mu \mathrm{m}$ is achieved using microscope objectives of NA $=0.5$ (similar to FF-OCT). Applied in the field of dermatology, the LF-OCT images reveal a comprehensive morphological mapping of skin tissues at a cellular level similar to histological images, in vivo (see Fig. 1). Using such a technology could help improve clinical diagnostic accuracy, allowing for the early detection of malignant skin tumors - including melanoma - and a reduction in the number of surgical excisions of benign lesions [9]. Other promising areas of application for LC-OCT include the imaging of lesions where conventional surgical excisions would be hazardous or impossible and the guidance of surgical interventions through identification of tumor margins. In addition, LC-OCT may also be used to identify the optimal area to biopsy in the case of extended lesions, thus reducing false negative rates from sampling errors.

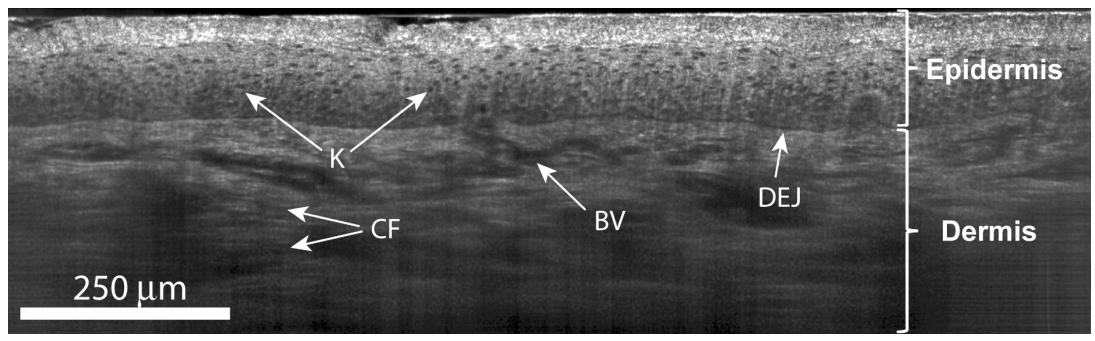

Fig. 1. LC-OCT image of human skin, in vivo, on the back of the hand. K: keratinocytes; DEJ: dermal-epidermal junction; CF: collagen fibers; BV: blood vessel.

\section{References}

[1] Dubois, A., Vabre, L., Boccara, A.C., Beaurepaire, E., "High-resolution full-field optical coherence tomography with a Linnik microscope," Appl. Opt. 41, 805-812 (2002).

[2] Vabre, L., Dubois, A., Boccara, A.C., "Thermal-light full-field optical coherence tomography," Opt. Lett. 27, 530-532 (2002).

[3] Dubois, A., Grieve, K., Moneron, G., Lecaque, R., Vabre, L., Boccara, A.C., "Ultrahigh-resolution full-field optical coherence tomography," Appl. Opt. 43, 2874-2882 (2004).

[4] Dubois, A., Moneron, G., Grieve, K., Boccara, A.C., "Three-dimensional cellular-level imaging using full-field optical coherence tomography," Phys. Med. Biol. 49, 1227-1234 (2004).

[5] Dubois, A., [Handbook of full-field optical coherence microscopy, technology and applications], Pan Stanford Publishing, Singapore, 1-51 (2016)

[6] Dubois A., "Focus defect and dispersion mismatch in full-field optical coherence microscopy," Appl. Opt. 57, 142-150 (2017).

[7] A. Dubois, O. Levecq, H. Azimani, A. Davis, J. Ogien, D. Siret, A. Barut, "Line-field confocal time-domain optical coherence tomography with dynamic focusing," Opt. Express 26, 33534-33542 (2018).

[8] Chen Y., Huang S.W., Aguirre A.D., Fujimoto J.G., "High-resolution line-scanning optical coherence microscopy," Opt. Lett. 32, 1971-1973 (2007).

[9] A. Dubois, O. Levecq, H. Azimani, D. Siret, A. Barut, M. Suppa, V. Del Marmol, J. Malvehy, E. Cinotti, J.L. Perrot, "Line-field confocal optical coherence tomography for high-resolution noninvasive imaging of skin tumors," J. Biomed. Opt. 23, 106007 (2018). 